\title{
Letter
}

\section{The great bustard reintroduction trial: a response to Ashbrook et al.}

The great bustard Otis tarda became extinct in the UK in the 183 os but has been the subject of a trial reintroduction on Salisbury Plain that began in 2004 (Osborne, 2005; Burnside et al., 2012; Ashbrook et al., 2016). Ashbrook et al. examined the long-term viability of this reintroduced population using data from the first 10 years of the reintroduction trial. They concluded that the outlook for the population was bleak and that it would decline unless substantial improvements could be made in survival rates and productivity.

However, any report written before the end of the trial reintroduction period, in March 2017, could not show the success that the project has achieved since 2014. The findings of Ashbrook et al. are predominantly for great bustards reintroduced from Russia and do not properly reflect the results achieved since great bustards reared in England from eggs imported from Spain have been released in the three breeding seasons from 2014 onwards.

Since sourcing birds from Spain rather than from Russia there have been many changes in rearing and release practices for the reintroduction. In particular, birds are no longer subjected to a month of pre-release quarantine, a necessary and understandable precautionary requirement for the import license provided by the Department for Environment, Food \& Rural Affairs. The mandatory design of the quarantine housing inhibited the pre-release fitness of the birds and their adaptation to their new habitat.

From 2014 onwards eggs have been imported exclusively from Spain and transported to a specialist incubation unit in England until hatching. Day-old chicks are reared on Salisbury Plain in secure pens that, without the restrictions imposed by regulations relevant to birds imported from outside the EU, better equip them for release. The birds are stronger and able to see the release area for a few months before their release into the wild. Supplementary feeding of the birds continues after release, enabling staff to monitor and identify the birds using a remote camera positioned by the feed trays.

Crucially, as the number of reintroduced birds has increased, there has been a more settled pattern of social behaviour that has led to greater site fidelity and lower overall mortality of new cohorts. This bodes well for higher reproduction rates, as well as for survival of wild-born chicks.

Not all previous wild eggs were predated, some just failed to hatch, most probably as a result of the immaturity of the males and females. In the 2016 breeding season a minimum of two wild-fledged chicks survived, one raised by a 2-yearold Spanish female. Females do not usually rear chicks successfully until their third year and have therefore not been factored into breeding projections.

A 5-year-old Russian female successfully fledged one chick in 2015 and another in 2016, and both chicks have survived. In 2016 there were a minimum of seven nesting attempts, five by 2-year-old Spanish females, which all produced hatched chicks.

In the 2016 season released chicks had an unprecedented $70 \%$ survival rate. Critically, there was no autumn dispersal of birds. Chicks, subadults and adults have formed male and female droves that, for the first time, replicate the natural behaviour of wild great bustard populations.

As a consequence, we are confident that the 2017 breeding season, with a greater number of older Spanish females in the population, will be even more productive. A full reintroduction programme, allowing significant releases for the next 3 years, should enable the numbers of the wild population to reach c. 100 and the great bustard will become re-established as a self-sustaining species in the UK. The full results of the trial reintroduction period (2004-2017) will be analysed and presented in due course.

Ruth MANVELL Great Bustard Group, Salisbury, UK

E-mail Ruth.Manvell321@btinternet.com

PAUL GoRIUP Fieldfare International, Newbury, UK

\section{References}

Ashbrook, K., Taylor, A., Jane, L., Carter, I. \& Székely, T. (2016) Impacts of survival and reproductive success on the long-term population viability of reintroduced great bustards Otis tarda in the UK. Oryx, 50, 583-592.

Burnside, R.J., Carter, I., Dawes, A., Waters, D., Lock, L., Goriup, P. \& Székely, T. (2012) The UK great bustard Otis tarda reintroduction trial: a 5-year progress report. Oryx, 46, 112-121.

Osborne, P.E. (2005) Key issues in assessing the feasibility of reintroducing the great bustard Otis tarda to Britain. Oryx, 39, 22-29. 